\title{
Postmortem prolactin as a marker of antemortem
}

\section{stress}

T J Jones, M J Hallworth

\begin{abstract}
Aim-To determine whether raised prolactin concentrations could be identified using postmortem venous blood and whether the level of prolactin correlated with antemortem stress.

Methods-Blood was obtained from the right femoral vein of 100 random adult necropsy cases, all of whom had been dead less than four days. Prolactin was determined in the samples by microparticle immunoassay. The levels of prolactin obtained were then analysed in relation to sex and cause of death, with particular emphasis on a history of antemortem stress and drug use.
\end{abstract}

Results-Prolactin in all cases of trauma was in the normal range (up to $500 \mathrm{mU} / \mathrm{l}$ ). In cases of sudden unexpected deaths the mean concentration was $533 \mathrm{mU} / 1$ (95\% confidence interval (CI), 372 to $694 \mathrm{mU} / 1)$. Postoperative deaths or cases with chronic disease had a mean value of $1027 \mathrm{mU} / 1$ (95\% CI, 735 to $1319 \mathrm{mU} / \mathrm{l})$. Cases of suicide had a mean value of $1398 \mathrm{mU} / 1$. Analysis of the suicides by sex showed a significant difference, the mean in female cases being $2072 \mathrm{mU} / 1$ compared with 692 mU/1 in male cases. In three of the four female suicides with the highest prolactin, the hyperprolactinaemia might have been attributable to a drug effect, but one case still had unexplained hyperprolactinaemia.

Conclusions-It is possible to detect prolactin reliably in postmortem venous blood samples. Prolactin values at necropsy differ according to the cause of death, with markedly higher values in postoperative deaths and in the chronically ill. Hyperprolactinaemia in cases of suicide is likely to result from the effects of the drugs used, but the levels were higher than previously reported.

Department of

Histopathology, Royal

Shrewsbury Hospital,

Mytton Oak Road,

Shrewsbury,

Shropshire SY3 8XQ, UK

$\mathrm{T}$ J Jones

Department of Biochemistry, Royal Shrewsbury Hospital M J Hallworth

Correspondence to: Dr Jones.

Accepted for publication 17 June 1999
Prolactin has a biological half life of $20 \mathrm{~min}$ utes but is stable in vitro for up to seven days at room temperature. No previous studies have been done to determine whether prolactin can be detected in postmortem blood samples.

The causes of hyperprolactinaemia are numerous. Physiological causes are pregnancy, breast feeding, nipple and breast stimulation, sleep, and exercise. Pathological causes ${ }^{1}$ include stress such as hypoglycaemia, surgery, myocardial infarction, and epilepsy; pituitary and hypothalamic disease interfering with central inhibitors of prolactin secretion; drugs, particularly dopamine blockers, methyldopa, oestrogens, opiates, ${ }^{2}$ tricyclic antidepressants, cimetidine, and verapamil. Some medical disorders such as hypothyroidism, chronic renal failure, and cirrhosis may also cause a marked increase in prolactin levels.

Previous studies have indicated a link between psychological stress and hyperprolactinaemia $^{3-8}$ but there have been no postmortem studies of cases of suicide to determine whether the antemortem psychological stress before self harm is associated with increased plasma prolactin at necropsy. No studies have been done to determine whether antemortem physical stress can be detected by raised postmortem prolactin.

The aims of this study were therefore to determine whether plasma prolactin could be measured in blood taken at necropsy, whether the level of prolactin correlated with a history of antemortem physical stress, and whether prolactin is increased in cases of fatal self harm. This would be useful if there was doubt about the interval between physical insult and death and in cases of suicide it would alert the investigator to the possibility of a drug overdose.

\section{Methods}

Blood was sampled from the right femoral vein at 100 adult necropsy examinations. Cases with known pituitary disease or with pituitary abnormalities found at necropsy were excluded. Before evisceration, an incision was made over the right femoral vein and the blood was removed by venepuncture. This was then analysed using an Abbott AxSym automated analyser. The working assay range was 15$4800 \mathrm{mU} / 1$ and no "high dose hook effect" occurs below $240000 \mathrm{mU} / 1 .{ }^{9}$ Dilution of serum samples showed acceptable parallelism. The assay was standardised against the WHO third international standard $84 / 500$. In studies performed by the kit manufacturer, the mean recovery of added prolactin from human serum was $95.4 \% .^{9}$

Full necropsy reports were available on all cases and these were then inspected for the antemortem clinical and drug history, 


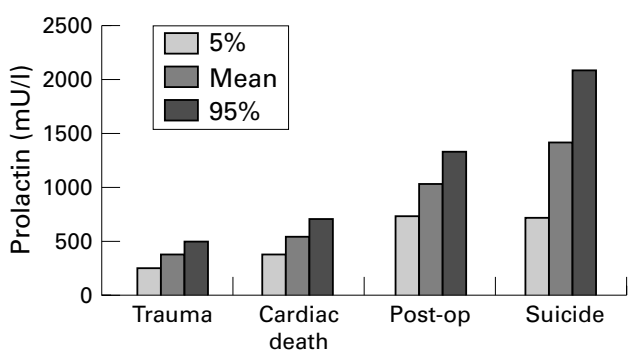

Figure 1 Prolactin concentration and cause of death.

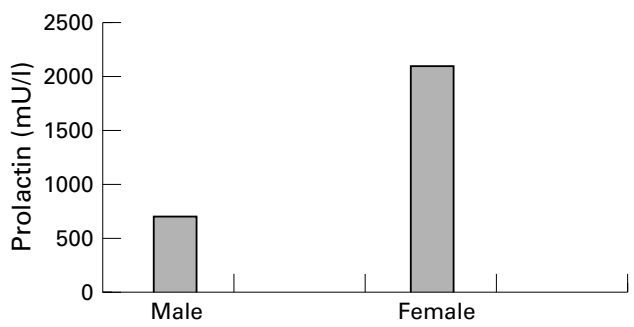

Figure 2 Prolactin concentrations in suicide cases by sex.

necropsy findings, and results of any toxicological analysis.

The cases sampled were then subdivided according to cause of death into traumatic deaths, sudden cardiac death, postoperative deaths and deaths from chronic medical conditions, and deaths from self harm.

Prolactin concentrations within each group were then analysed statistically to determine whether any significant differences were identifiable. The self harm group was analysed according to sex and manner of death.

\section{Results}

Six of the 100 samples were haemolysed and thus unsuitable for analysis. Of the remaining 94 cases, 17 died from trauma, 19 were sudden cardiac deaths, 42 were postoperative or caused by chronic medical conditions, and 16 were suicides. Figure 1 shows the prolactin concentrations in relation to the cause of death.

The mean prolactin in cases of suicide was $1398 \mathrm{mU} / 1$ (normal up to $500 \mathrm{mU} / \mathrm{l}$ ) compared with $777 \mathrm{mU} / 1$ in the non-suicides. This difference was statistically significant $(p=0.0140)$.

In all cases of death from trauma prolactin was within the normal range. Analysis of the necropsy reports in these cases showed that death occurred at or very soon after the traumatic event

In sudden unexpected deaths from diseases of the cardiovascular system mean prolactin concentration was just above normal (533 $\mathrm{mU} / \mathrm{l} ; 95 \%$ confidence interval (CI), 372 to $694 \mathrm{mU} / \mathrm{l}$ ).

Mean prolactin in postoperative deaths and those from chronic disease was clearly raised (1027 mU/1; 95\% CI, 735 to $1319 \mathrm{mU} / \mathrm{l}$ ).

Analysis of prolactin level by sex in suicide cases showed that female victims had a mean value of $2072 \mathrm{mU} / 1$ compared with $692 \mathrm{mU} / \mathrm{l}$ in male victims; this difference was significant ( $\mathrm{p}<0.05)$ (fig 2).

The causes of death in female suicide cases and their prolactin levels are shown in table 1 . The highest levels of prolactin were found in
Table 1 Cause of death in female cases of suicide and level of prolactin

\begin{tabular}{ll}
\hline Cause of death & $\begin{array}{l}\text { Prolactin } \\
(\mathrm{mU} / \mathrm{l})\end{array}$ \\
\hline Co-proxamol and dihydrocodeine overdose & 4034 \\
Paracetamol overdose & 1813 \\
Co-proxamol overdose & 2341 \\
CO poisoning & 2852 \\
Hanging & 3981 \\
Hanging & 809 \\
Hanging & 462 \\
Hanging & 283 \\
\hline
\end{tabular}

drug related deaths; the case of hanging with a level of $3981 \mathrm{mU} / 1$ was an exception, although in this case the woman was taking hormone replacement therapy. The three other cases of female hanging had a mean prolactin concentration of $518 \mathrm{mU} / 1$.

The remaining female suicide case died of carbon monoxide poisoning and had a prolactin concentration of $2852 \mathrm{mU} / 1$. This could not be attributed to any identifiable drug effect.

\section{Discussion}

In this study we showed that it is possible to measure prolactin in postmortem venous blood and to identify hyperprolactinaemia.

The level of prolactin found was roughly comparable with the duration of antemortem physical stress, cases of death from trauma, where death was very rapid, having a normal prolactin level. With sudden cardiac events, where death is often not so rapid, the mean prolactin was just above normal, presumably because in some cases the delay between onset of symptoms and death has allowed a stress response to develop. The postoperative deaths and those from chronic medical conditions had a mean value well above normal, indicating that in this group a stress response was well developed, with a consistently raised prolactin.

Some cases of suicide have significantly raised values, although on further analysis this may be attributable to drug effects (for example, codeine, dextropropoxyphene, or paracetamol induced liver necrosis), although the levels found in this study were greater than those previously described in living volunteers. ${ }^{2}$ In addition, one individual had hyperprolactinaemia that could not be attributable to any known drug effect.

There is therefore some support in this study for the hypothesis that antemortem psychological stress such as depression produces hyperprolactinaemia identifiable postmortem. Cases with physical stress had a raised prolactin and this may therefore be a useful adjunct to forensic investigation when the possible length of survival after a traumatic event is unclear.

1 Molitch ME. Pathologic hyperprolactinaemia. Endocrinol Metab Clin North Am 1992;21:877-901.

2 Zis AP, Haskett RF, Albala AA, et al. Morphine inhibits cortisol and stimulates prolactin secretion in man. Psychoneuroendocrinology 1984;9:423-7.

3 Reavley A, Fisher AD, Owen D, et al. Psychological distress in patients with hyperprolactinaemia. Clin Endocrinol 1997; in patients

4 Fava M, Serafini E, De Besi L, et al. Hyperprolactinaemia and psychological distress in women undergoing chronic haemodialysis. Psychother Psychosom 1988;49:6-9. 
5 Kellner R, Buckman MT, Fava GA, et al. Hyperprolactinaemia, distress and hostility. Am $\mathcal{F}$ Psychiatry 1984;141:759mia,

6 Fava M, Fava GA, Kellner R, et al. Psychosomatic aspects of

hyperprolactinaemia. Psychother Psychosom 1983;40:257-62.

7 Fava M, Fava GA, Kellner R, et al. Depression and hostility in hyperprolactinaemia. Prog Neuropsychopharmacol Biol Psychiatry 1982;6:479-82.

8 Merritt D F. Hyperprolactinaemia and depression. $7 A M A$ 1991;266:2004.

9 Abbott Laboratories, Diagnostics Division. Prolactin (package insert). Abbott Park, IL: Abbott Laboratories, 1995.

\section{Royal College of Pathologists}

Forthcoming symposia to be held at 2 Carlton House Terrace, London SW1:

Pulmonary pathology: 7 October 1999

Understanding stillbirth-is there progress?: 20 October 1999

Genomics in therapy and xenotransplantation: safety and ethics: 24 November 1999

Cytopathology update. Searching for cancer: 14 December 1999

Can't give a cause of death-won't give a cause of death: 12 January 2000

Molecular genetics of solid tumours: translating research into clinical practice:

24 February 2000

Managing immunodeficiency: 22 March 2000

Histopathology update: GI and hepatobiliary update and controversies:

13-14 April 2000

These meetings are open to members and non-members of the college. Further details and application forms from:

Scientific Meetings Officer, RCPath, 2 Carlton House Terrace, London SW1Y 5AF, UK; tel +44 (0)171930 5862, ext 24/25; fax +44(0)1713210523. 